\title{
Correlation between ETFDH mutations and dysregulation of serum myomiRs in MADD patients
}

\author{
Sara Missaglia $\left(1,2^{*}\right)$, Valentina Pegoraro (3*), Roberta Marozzo (3), Daniela Tavian \\ $(1,2)$, Corrado Angelini (3) \\ (1) Laboratory of Cellular Biochemistry and Molecular Biology, CRIBENS, Università Cattolica \\ del Sacro Cuore, Milan, Italy; (2) Department of Psychology, Università Cattolica del Sacro \\ Cuore, Milan, Italy; (3) IRCCS San Camillo Hospital, Venice, Italy \\ *Both these authors contributed equally to this work and should be considered as first authors \\ This article is distributed under the terms of the Creative Commons Attribution Noncommercial License (CC BY-NC 4.0) which permits
} any noncommercial use, distribution, and reproduction in any medium, provided the original author(s) and source are credited.

\begin{abstract}
Multiple acyl-CoA dehydrogenase deficiency (MADD) is a rare fatty acids oxidation disorder which is often associated with deficiency of electron transfer flavoprotein dehydrogenase (ETFDH). In this study we reported clinical features and evaluation of expression profile of circulating muscle-specific miRNAs (myomiRs) in two MADD patients carrying different ETFDH gene mutations. Patient 1 was a compound heterozygote for two missense mutations. She showed a late onset MADD clinical phenotype and a significant increase of serum myomiRs. Patient 2, carrying a missense and a frameshift mutation, displayed early onset symptoms and a slight increase of some serum myomiRs.
\end{abstract}

Key Words: Multiple acyl-CoA dehydrogenase deficiency, fatty acids oxidation disorder, myopathy, ETFDH, myomiRs.

Deficiency of some enzymes involved in the fatty acids (FA) oxidation results in an impairment of FA breakdown. An abnormal storage of lipids, especially in skeletal muscle fibers, has been observed in several FA oxidation disorders. ${ }^{1}$ Multiple acyl-coenzyme A dehydrogenase deficiency (MADD) is a rare autosomal recessive defect of mitochondrial FA oxidation. MADD is due to mutations in several genes. The majority of patients carries mutations in ETFDH gene. ${ }^{2}$ ETFDH encodes electron transfer flavoprotein dehydrogenase (ETFDH), a $\beta$-oxidation enzyme which is involved in the electron transfer of respiratory chain. This protein has three main functional domains: FAD-binding domain, $4 \mathrm{Fe} 4 \mathrm{~S}$ cluster and ubiquinone (UQ) binding domain. ${ }^{3}$ Generally, mutations of ETFDH are associated with late onset MADD. Clinical symptoms are highly variable, and their severity seems to correlate with localization of mutations in different protein regions. ${ }^{2}$ The patients might present recurrent episodes of vomiting, metabolic acidosis, lethargy and muscle injury (proximal myopathy, myalgia and muscle weakness). It is important to evaluate clinical symptoms progression and risk factors involved in the evolution of myopathy. A massive excretion of several organic acids (glutaric, lactic, ethylmalonic, butyric, isobutyric, 2-methylbutyric and isovaleric acids) occurs in these subjects. ${ }^{4}$ Therapy with supplementation of riboflavin, L-carnitine and/or coenzyme Q10 and a special diet (poor in fat and protein) can determine a significant improvement of clinical conditions. ${ }^{2}$ Biochemical and bioinformatic analysis are available to predict the pathological effect of ETFDH mutations on protein function, and there is a genotypephenotype correlation. Moreover, the use of muscle imaging analysis (i.e CT scan and MRI) provides information on muscle damage progression. ${ }^{5,6}$ Several studies report the central role of small, non-coding RNA (called miRNAs) in the regulation of gene expression of many biological processes, including myogenesis. ${ }^{7} \mathrm{~A}$ particular class of miRNAs, named myomiRs, includes molecules expressed both in skeletal and cardiac muscle. ${ }^{8}$ MyomiRs can be secreted by muscle fibers in serum by an active or passive process and they are called circulating myomiRs. MyomiR secretory activity during physical exercise or in neuromuscular disorders utilizes extracellular vesicles, microvesicles exosomes, highdensity lipoprotein. On the contrary, a passive release takes place as a result of myofiber necrosis (Figure 1a) ${ }^{8,9}$ Canonical muscle-specific miRNAs are: miR-1, miR133a, miR-133b and miR-206. They show different expression profile in skeletal and cardiac muscle. ${ }^{10}$ There 
ETFDH mutations and dysregulation of serum myomiRs in MADD patients

a

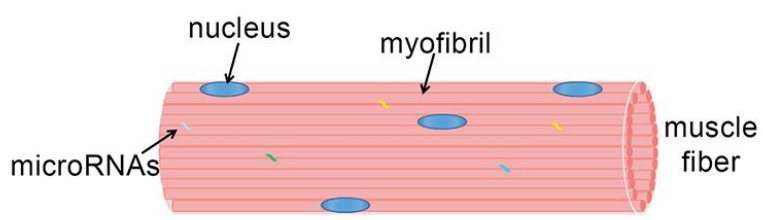

Active secretion during exercise or neuromuscular disorders
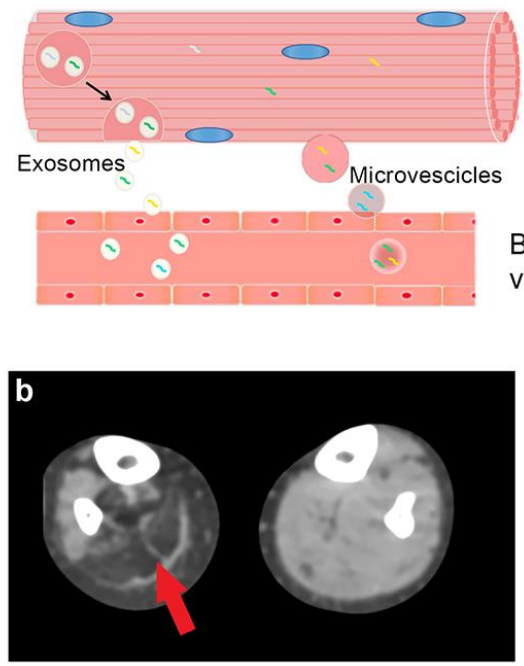

d

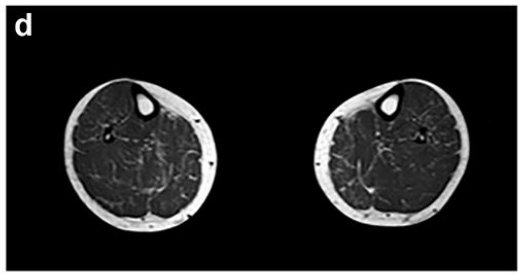

Passive release in case of fiber necrosis

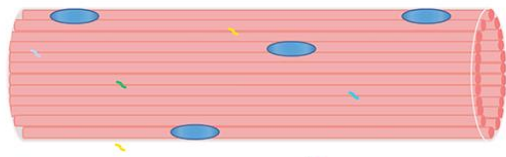

Blood vessel
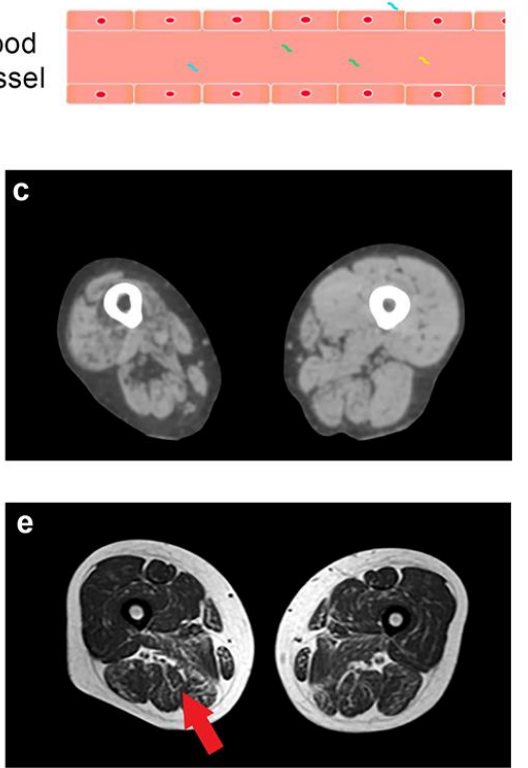

Fig. 1. MyomiRs secretion or passive release from skeletal muscle fibers to circulatory blood flow. In muscular disorders or during exercise an active secretion of myomiRs might occur through the formation of exosomes or microvesicles releasing the myomiRs in the blood. If myofiber necrosis occurs, myomiRs can be secreted directly in the blood for plasma membrane defects $(\boldsymbol{a})$. Evaluation of fibro-fatty replacement in lower limbs of two MADD patients. CT scan of patient 1 showed marked fibro flatty replacement in one leg. Mercuri score of soleus and gastrocnemius muscles (red arrow) is 4 while the other leg muscles are spared (b). In thigh muscles, marked atrophy was observed, especially in posterior compartment and slight involvement on the other side (c). Muscle MRI of patient 2 showed no evident fatty infiltrations in leg muscle (d). Muscle atrophy and slight alteration of posterior thigh muscles was observed, i.e. semimembranosus and adductor magnus (red arrow) with fibro fatty replacement $(\boldsymbol{e})$.

are evidences that miR-1, miR-133a and miR-133b are present in both muscle types, while miR-206 is specifically expressed in skeletal muscle. miR-1 and miR-206 regulate myogenic differentiation, while miR$133 \mathrm{a}$ and miR-133b stimulate cell proliferation. ${ }^{11}$ Changes of serum myomiRs levels can be related to progression of the disease, the evaluation of myomiRs expression profile could be an important tool to verify progression of muscle damage. ${ }^{12,13}$ In this study we describe the correlation between clinical phenotype, and expression profile of circulating myomiRs in two unrelated MADD patients.

\section{Materials and Methods}

This study involves two MADD patients, which have previously described. ${ }^{14,15}$ Muscle examination was carried out using muscle computed tomography (CT) scan and muscle magnetic resonance imaging (MRI). Muscle pattern was ranked according to Mercuri scale. All procedures were approved by the local Institutional Review Board (IRB) committee and were conducted in 
accordance with the ethical principles outlined in the Declaration of Helsinki. All patients signed a written, informed consent. Some bioinformatic software (ClustalW, SIFT, PolyPhen and I-Tasser) were used to analyze the effect of mutations on ETFDH protein function and structure. The NCBI reference sequence (NP_001268666.1) of the human ETFDH protein was used as the input for protein function investigation, while the porcine ETFDH structure, which has been obtained from x-ray crystallography analysis and shares $95 \%$ of homology with human aa sequence, was used to generate protein 3D models. After treatment, serum samples were obtained from both patients. Total RNA, including miRNAs, was obtained from $400 \mu$ l of serum using the miRNeasy Mini Kit (QIAzol). Expression profile of miRNAs was verified by real-time polymerase chain reaction (qRT-PCR), performed using $10 \mathrm{ng}$ RNA as template and the CFX96 ${ }^{\mathrm{TM}}$ Real-Time PCR detection System (Bio-Rad), with specific TaqMan MicroRNA Assay. The level of each miRNA was normalized to miR16, U6 snRNA, as internal controls, and miR-39-3p of Caenorhabditis elegans, as spike-in miRNA. The Wilcoxon-Mann-Whitney test was used to determine statistical significance which was defined as ${ }^{*} P<0.05$. All studies were done in three separate experiments, each performed in triplicate. All data were expressed as the mean \pm standard deviation.

\section{Results and Discussion}

Patient 1, 61-year old woman, presented a late onset of MADD. In particular, skeletal and respiratory muscle weakness occurred at 38 years. She was a compound heterozygote, carrying two missense mutations in the ETFDH gene (c.560C $>$ T [p.A187V] and c.1027T $>C$ [p.W343R]). Patient 2, now 54 years old, showed her first symptoms, i.e. recurrent episodes of vomiting, drowsiness, appetite loss, asthenia and acetonemic breath, at 2 years of age and a lipid storage myopathy at 6 years of age. In this patient, genetic analysis revealed the c.560C>T (p.A187V) and the c.1285+1G>A (p.G429Dfs21*) ETFDH gene mutations (a missense and a splice site variant, respectively). Muscle evaluation showed type I vacuolated fibers in patient $2,{ }^{15}$ and muscle atrophy in both patients. Moreover, CT scan and MRI displayed alterations of posterior thigh muscles (Figure 1b-e). Both patients followed a low-fat, high-protein diet, supplemented with medium-chain triglyceride (MCT) oil, L-carnitine and riboflavin. This treatment decreased metabolic crisis and improved muscle injury. To elucidate the pathogenetic impact of mutations on ETFDH structure and activity, bioinformatic investigations were performed. ClustalW analysis system showed that both amino acids, A187 and W343, were localized in two highly conserved domains of ETFDH protein, thus indicating that they could affect enzyme function. This hypothesis was reinforced by PolyPhen-2 and SIFT analysis, which predicted a deleterious effect of both mutations. In particular, PolyPhen-2 indicated a
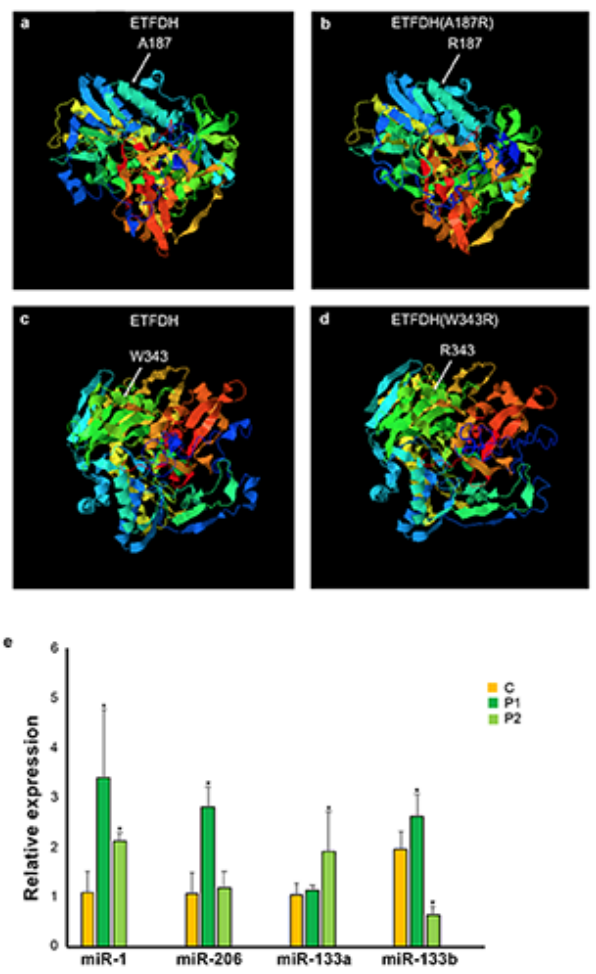

Fig. 2. Bioinformatic analysis of ETFDH mutant protein and evaluation of circulating myomiRs expression profile. $\boldsymbol{a}, \boldsymbol{b} 3 D$ structure of ETFDH(A187V), compared to ETFDH wild type. The p.A187V shows severe modifications of protein folding; $\boldsymbol{c}, \boldsymbol{d}$ ETFDH(W343R) tridimensional model reveals partial alterations of enzyme structure, in comparison with native ETFDH; $\boldsymbol{e}$ Evaluation of serum myomiRs in MADD patients, compared to a control group. The bar graph representsmean values of circulating myomiRs. Control value is set to $1 .{ }^{*} P \leq .05\left(^{*}\right)$. C: pool of controls; P1: MADD patient 1; P2: MADD patient 2.

score of 1 (score from 0 to $1, \geq 0.8$ is probably damaging) and SIFT a score of 0 (score from 1 to $0, \leq 0.05$ is damaging). To verify whether the missense mutations altered protein structure, 3D models of ETFDH mutant proteins were obtained using I-Tasser software. ETFDH(A187V) was predicted to cause changes of secondary structure in the FAD-binding region, determining its incorrect folding (Figure $2 \mathrm{a}$ and $\mathrm{b}$ ). The tridimensional structure prediction analysis of ETFDH(W343R) revealed a modification of the secondary structure in some amino acids located in the 4Fe4S cluster and in the UQ-binding domain (Figure 2c and $d)$. These alterations could only partially affect ETFDH conformation. 
In patient 1 , who carried two missense mutations, a late onset of MADD was diagnosed. Both these variants are predicted to determine an alteration of ETFDH folding. However, while the p.A187V modifies the FAD-binding domain, decreasing its capacity to interact with $\mathrm{FAD}^{16}$, the p.W343R missense variant behaves differently.

The A187V mutation, in the first allele of patient 1 , might result in a less stable protein than that produced by the second allele, carrying the W343R variation. Patient 2 carried out the p.A187V and p.G429Dfs21* mutations. p.A187V might cause a strong reduction of ETFDH function. The frameshift mutation is likely going to cause nonsense mediated mRNA decay so no protein is probably made by this allele. ${ }^{17}$ Even if there is some residual mRNA the resulting protein is going to be unstable and rapidly degraded. The ETFDH genetic profile of this patient could be more compromised than in the first patient and this might explain the early onset and clinical symptoms. The evaluation of serum myomiRs profiles in MADD patients showed a dysregulation in both subjects (Figure 2e). Patient 1 had an upregulation of miR-1, miR-206 and miR-133b; while miR-133a had an expression level similar to control. In patient 2, an increase of miR-1 and miR-133a was observed, while miR-206 had normal expression level and miR-133b was downregulated. MyomiRs act as muscle regulators and their balance maintains muscle homeostasis under normal cellular conditions. ${ }^{11} \mathrm{MiR}-206$ is implicated in muscle regeneration and maturation, and in skeletal muscle mass regulation. ${ }^{10}$ miR-1 and miR-206 regulate myogenic differentiation, while miR-133a and miR-133b preserve cell undifferentiated state and stimulate muscle growth. ${ }^{18}$ In patient 1 , the upregulation of miR-1, miR-206 and miR-133a suggests that muscle regeneration might be stimulated in this subject. In patient 2, with severe impairment of ETFDH activity in comparison with the first patient, the decrease of serum miR-133b amount and the normal levels of circulating miR-206 indicates less active regeneration.

In conclusion, we report bioinformatic and serum myomiRs investigations in two MADD patients. Missense mutations may cause different degrees of ETFDH function impairment. The severity of MADD clinical phenotype, in particular marked atrophy of some muscles (i.e. posterior thigh muscle in both patients, soleus and gastrocnemius muscles in the leg of patient 1 ), is correlated with the decrease of ETFDH protein function and with dysregulation of circulating myomiRs expression profile. We suggest that in MADD, as well as in other muscle disorders, the level of serum myomiRs might represent a biomarker of skeletal muscle involvement.

\section{List of acronyms}

FA, fatty acids

MADD, Multiple acyl-coenzyme A dehydrogenase deficiency

ETFDH, electron transfer flavoprotein dehydrogenase
myomiRs, muscle-specific miRNA

CT, computed tomography scan

MRI, muscle magnetic resonance imaging qRT-PCR, real-time polymerase chain reaction MCT, medium-chain triglyceride

\section{Authors contributions}

SM wrote and edited the manuscript; VP performed myomiRs analysis; RM performed statistical analysis; DT critically revised the manuscript; CA conceived the study, supervised it and performed the clinical characterization of patients. All authors read and approved the final manuscript.

\section{Acknowledgments}

The authors thank all patients and volunteers involved in the study and the Biobank BBMRNR of IRCCS San Camillo Hospital for providing biological samples.

\section{Funding}

The publication of this article was supported by Università Cattolica del Sacro Cuore (Milan, Italy). This work was supported by PRIN Grant 2017A5TXC3 (Italy) and AFM grant 22392 (France).

\section{Conflict of Interest}

The authors declare they have no financial, personal, or other conflicts of interest.

\section{Ethical Publication Statement}

We confirm that we have read the Journal's position on issues involved in ethical publication and affirm that this report is consistent with those guidelines.

\section{Corresponding Author}

Corrado Angelini, IRCCS San Camillo Hospital, via Alberoni 70, 30126 Venezia, Italy

ORCID iD: http://orcid.org/0000-0002-9554-8794

Email: corrado.angelini@ospedalesancamillo.net

Email of co-authors

SaraMissaglia: sara.missaglia@unicatt.it ORCID iD: http://orcid.org/0000-0001-6551-6698

Valentina Pegoraro: valentina.pegoraro@ospedalesancamillo.net ORCID iD: http://orcid.org/0000-0002-7396-3022

Roberta Marozzo: roberta.marozzo@ospedalesancamillo.net ORCID iD: http://orcid.org/0000-0001-7830-3456

Daniela Tavian: daniela.tavian@unicatt.it ORCID iD: http://orcid.org/0000-0003-3333-0068

\section{References}

1. Merritt JL 2nd, Norris M, Kanungo S. Fatty acid oxidation disorders. Ann Transl Med 2018;6:473.

2. Angelini C, Pennisi E, Missaglia S, et al. Metabolic lipid muscle disorders: biomarkers 


\section{ETFDH mutations and dysregulation of serum myomiRs in MADD patients}

Eur J Transl Myol 30 (1): xx1-xx5, 2020

and treatment. Ther Adv Neurol Disord 2019;12:1756286419843359.

3. Zhang J, Frerman FE, Kim JJ. Structure of electron transfer flavoprotein-ubiquinone oxidoreductase and electron transfer to the mitochondrial ubiquinone pool. Proc Natl Acad Sci U S A 2006;103:16212-7.

4. Grunert SC. Clinical and genetical heterogeneity of late-onset multiple acylcoenzyme a dehydrogenase deficiency. Orphanet J Rare Dis 2014;9:117.

5. Edmunds KJ, Gíslason MK, Arnadottir ID, et al. Quantitative Computed Tomography and Image Analysis for Advanced Muscle Assessment. Eur J Transl Myol 2016;26:6015.

6. Gargiulo P, Helgason T, Ramon C, Jr HJ, Carraro U. CT and MRI Assessment and Characterization Using Segmentation and 3D Modeling Techniques: Applications to Muscle, Bone and Brain. Eur J Transl Myol 2014;24:3298.

7. Bhaskaran M, Mohan M. MicroRNAs: History, Biogenesis, and Their Evolving Role in Animal Development and Disease. Vet Pathol 2014;5:759-74.

8. Siracusa J, Koulmann N, Banzet S. Circulating myomiRs: a new class of biomarkers to monitor skeletal muscle in physiology and medicine. $\mathbf{J}$ Cachexia Sarcopenia Muscle 2018;9:20-27.

9. Vickers KC, Palmisano BT, Shoucri BM, et al. MicroRNAs are transported in plasma and delivered to recipient cells by high-density lipoprotein. Nat Cell Biol 2011;13:423-433.

10. Horak $M$, Novak J, Bienertova-Vasku J. Muscle-specific microRNAs in skeletal muscle development. Dev Biol 2016;410:1-13.

11. Mitchelson KR, Qin WY. Roles of the canonical myomiRs miR-1, -133 and -206 in cell development and disease. World J Biol Chem 2015;6:162-208.

12. Cacchiarelli D, Legnini I, Martone J, et al. miRNAs as serum biomarkers for Duchenne muscular dystrophy. EMBO Mol Med 2011;3:258-65.

13. Pegoraro V, Missaglia S, Marozzo R, et al. MiRNAs as biomarkers of phenotype in neutral lipid storage disease with myopathy. Muscle Nerve 2020;61:253-7.

14. Angelini C, Tavian D, Missaglia S. Heterogeneous Phenotypes in Lipid Storage Myopathy Due to ETFDH Gene Mutations. JIMD Rep 2018;38:33-40.

15. Missaglia S, Tavian D, Moro L, et al. Characterization of two ETFDH mutations in a novel case of riboflavin-responsive multiple acyl-CoA dehydrogenase deficiency. Lipids Health Dis 2018;17:254.

16. Macchione F, Salviati L, Bordugo A, et al. Multiple acyl-COA dehydrogenase deficiency in elderly carriers. J Neurol 2020 Jan 29. doi: 10.1007/s00415-020-09729-z

17. Karamyshev AL, Karamysheva ZN. Lost in Translation: RibosomeAssociated mRNA and Protein Quality Controls. Front Genet 2018;9:431.

18. Townley-Tilson WHD, Callis TE, Wang D. MicroRNAs 1, 133, and 206: Critical factors of skeletal and cardiac muscle development, function, and disease. Int $\mathbf{J}$ Biochem Cell Biol 2010;42:1252-5.

Submissions: Febraury 05, 2020

Revision received: Febraury 13, 2020

Acceptance: Febraury 15, 2020 